\title{
Developmental Switch in the Short-Term Modification of Unitary EPSPs Evoked in Layer 2/3 and Layer 5 Pyramidal Neurons of Rat Neocortex
}

\author{
Alex Reyes and Bert Sakmann \\ Abteilung Zellphysiologie, Max-Planck-Institut für medizinische Forschung, D-69120 Heidelberg, Germany
}

Amplitudes of EPSPs evoked by repetitive presynaptic action potentials can either decrease (synaptic depression) or increase (synaptic facilitation). To determine whether facilitation and depression in the connections between neocortical pyramidal cells varied with the identity of the pre- or the postsynaptic cell and whether they changed during postnatal development, whole-cell voltage recordings were made simultaneously from two or three pyramidal cells in layers $2 / 3$ and 5 of the rat sensorimotor cortex. Unitary EPSPs were evoked when preand postsynaptic neurons were in the same and in different layers. In young [postnatal day 14 (P14)] cortex, EPSPs evoked in all connected neurons depressed. The degree of depression was layer specific and was determined by the identity of the presynaptic cell. EPSPs evoked by stimulation of presynaptic layer 5 neurons depressed significantly more than did those

The establishment of representational maps in the mammalian cortex requires highly specific formation and elimination of synaptic contacts between neurons located in the same and in different cortical layers (Goodman and Shatz, 1993; Katz and Shatz, 1996). Both the stabilization and elimination of synapses during cortical development are thought to depend, in part, on the electrical activity of thalamocortical and intracortical connections (Katz and Shatz, 1996). The efficacy of excitatory synaptic transmission between pyramidal neurons, the major cell class of the cortex, varies with the level of neuronal activity. When a presynaptic cell discharges repetitively, the amplitude of EPSPs evoked in the target cells can decrease (depress) or increase (facilitate) on a relatively short timescale (Thomson et al., 1993; Thomson and Deuchars, 1994; Markram and Tsodyks, 1996; Stratford et al., 1996; Abbott et al., 1997; Buhl et al., 1997; Thomson, 1997; Ali and Thomson, 1998; Ali et al., 1998). Therefore, short-term modification of EPSPs could determine the spread of electrical activity in and between cortical layers and influence, during development, the establishment of specific synaptic connections.

To elucidate synaptic mechanisms that may contribute to activity-driven formation of cortical connections, we determined the short-term modification of EPSPs in pyramid-to-pyramid connections within and between two cortical layers at different stages of postnatal development. We recorded simultaneously from two or three synaptically connected pyramidal cells located in layer

\footnotetext{
Received Oct. 28, 1998; revised Feb. 23, 1999; accepted Feb. 25, 1999.

A.R. was supported by the Alexander von Humboldt Stiftung and the Max-Planck Gesellschaft. We thank Dr. G. G. Borst for comments on this manuscript.

Correspondence should be addressed to Dr. Alex Reyes, Center for Neural Science, Room 809, New York University, New York, NY 10003.

Copyright (C) 1999 Society for Neuroscience 0270-6474/99/193827-09\$05.00/0
}

evoked by stimulation of layer $2 / 3$ neurons. In mature cortex (P28), however, the EPSPs evoked in these connected neurons facilitated to a comparable degree regardless of the layer in which pre- and postsynaptic neurons were located. The results suggest that in young cortex the degree of synaptic depression in connected pyramidal cells is determined primarily by whether the presynaptic cell was in layer $2 / 3$ or 5 and that maturation of the cortex involves a developmental switch from depression to facilitation between P14 and P28 that eliminates the layerspecific differences. A functional consequence of this switch is that in mature cortex the spread of excitation between neocortical pyramidal neurons is enhanced when action potentials occur in bursts.

Key words: neocortex; development; facilitation; depression; synapses; postsynaptic potentials
$2 / 3$ and layer 5 and measured the short-term modification of unitary EPSPs. Via the use of triple recordings, we show that the EPSPs evoked in neurons in young [postnatal day 14 (P14)] cortex all depressed, the degree of which was layer specific and determined by the location of the presynaptic cells. These differences in short-term modification were absent in mature (P28) cortex. The EPSPs evoked in pyramidal cells at both layers facilitated. These changes may be functionally important for the spread of activity in the neocortex between and within cortical layers during development.

\section{MATERIALS AND METHODS}

Preparation and recording. Slices were prepared as described in Stuart et al. (1993). During recordings, slices were maintained at $34^{\circ} \mathrm{C}$ in artificial CSF consisting of (in mM): $125 \mathrm{NaCl}, 2.5 \mathrm{KCl}, 25$ glucose, $25 \mathrm{NaHCO}_{3}$, $1.25 \mathrm{NaH}_{2} \mathrm{PO}_{4}, 2 \mathrm{CaCl}_{2}$, and $1 \mathrm{MgCl}_{2}$. Individual cells were visualized using infrared differential interference contrast (IR-DIC) microscopy (Stuart et al., 1993). The water immersion objective had a $40 \times$ magnification. Two CCD cameras were mounted on a beam splitter so that the slice could be viewed at two different magnifications. Pyramidal neurons were identified under IR-DIC video microscopy by their location in the slice and by their distinct apical dendrites that extended toward the pial surface. Whole-cell voltage recordings were made simultaneously from three neurons using pipettes with 5-15 $\mathrm{M} \Omega$ resistance when filled with (in $\mathrm{mM}$ ): $100 \mathrm{~K}$-gluconate, $20 \mathrm{KCl}, 4 \mathrm{ATP}-\mathrm{Mg}, 10$ phosphocreatine, 0.3 GTP, and 10 HEPES, pH 7.3 (310 mOsm). Recordings were performed in current clamp using Axoclamp 2B amplifiers (Axon Instruments, Foster City, CA). In synaptically connected neurons, suprathreshold stimulation of the presynaptic cells evoked unitary EPSPs in the target cell(s). Presynaptic cells were stimulated by delivering two to three current pulses $(5 \mathrm{msec})$ at $10-80 \mathrm{~Hz}$. Most of the data were taken with $10 \mathrm{~Hz}$ stimulation to prevent the long-term changes in EPSP amplitudes often associated with higher frequency stimulation and to minimize temporal summation of the EPSPs. Trains of stimuli were separated by 


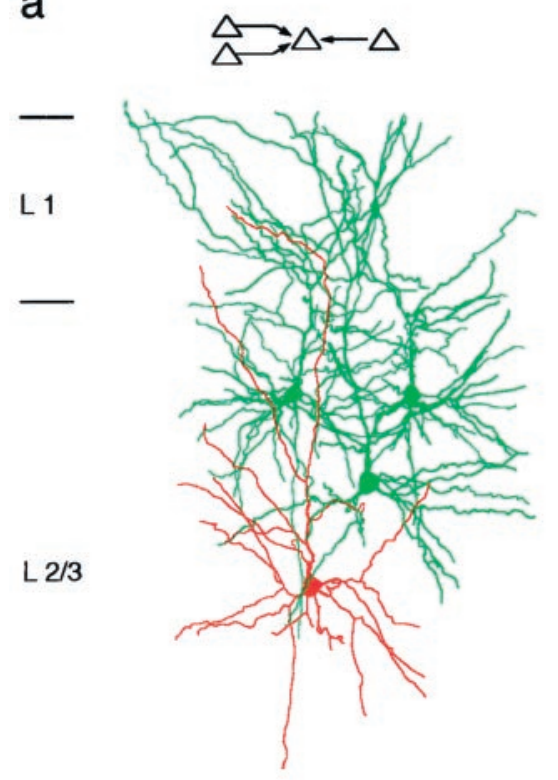

Figure 1. Unitary EPSPs evoked in connected layer $2 / 3$ and in connected layer 5 neocortical pyramidal neurons. $a$, Camera lucida reconstructions of pyramidal neurons in layer 2/3 from a P14 rat. Three cells (green) innervated a fourth pyramidal cell (red), as indicated in the schematic drawing (inset). Neurons were filled with biocytin during the experiment. Only somata and dendritic arbors were reconstructed. Calibration bar, $100 \mu \mathrm{m}$. $b$, Camera lucida reconstructions of three layer 5 pyramidal neurons from a P14 rat. Two neurons (green) innervated the same postsynaptic neuron (red). In addition, one of the neurons simultaneously innervated two neurons [schematic drawing (inset)]. Calibration bar, $100 \mu \mathrm{m}$. $c$, Unitary EPSPs recorded in the same layer $2 / 3$ pyramidal cell during $10 \mathrm{~Hz}$ stimulation of three different presynaptic pyramidal cells. Numbered tic marks in the upper trace give the times of occurrences of the presynaptic action potentials. The three lower traces represent averages of EPSPs compiled from 50 to 100 sweeps. The resting membrane potential of the postsynaptic cell was $-69 \mathrm{mV}$. Recordings were obtained from the neurons shown in $a . d$, Unitary EPSPs recorded in two layer 5 neurons during $10 \mathrm{~Hz}$ stimulation of the presynaptic neurons. The circuit is depicted in the inset of $b$. The timing of presynaptic action potentials is shown in the upper trace. The second and fourth traces from the top show EPSPs evoked in a common target neuron during stimulation of two presynaptic neurons. The second and third traces show EPSPs evoked in two different pyramidal neurons during stimulation of a single presynaptic pyramidal neuron. Resting membrane potentials of the two postsynaptic cells were -62 and $-64 \mathrm{mV}$.e, Summary of the short-term modification of EPSPs evoked in layer $2 / 3$ pyramidal neurons expressed as the ratio of the amplitude of the second EPSP to that of the first EPSP $($ EPSP2/EPSP1 $\times 100)$. Connected squares represent the amplitude ratios for EPSPs evoked in a common neuron during $10 \mathrm{~Hz}$ stimulation of two or more presynaptic pyramidal neurons. The mean $( \pm$ SD) amplitude ratio of EPSPs (shown on the right) was $89 \pm 17 \%(n=16) . f$, Summary of the amplitude ratios of EPSPs evoked in the same layer 5 neuron during $10 \mathrm{~Hz}$ stimulation of two presynaptic layer 5 neurons. The mean $( \pm \mathrm{SD})$ amplitude ratio (shown on the right) was $69 \pm 19 \%$ $(n=16)$. a

b

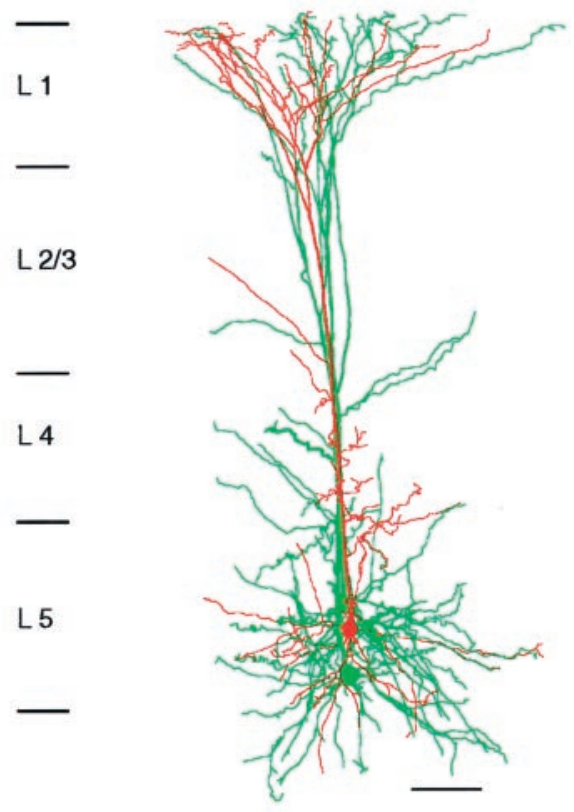

C

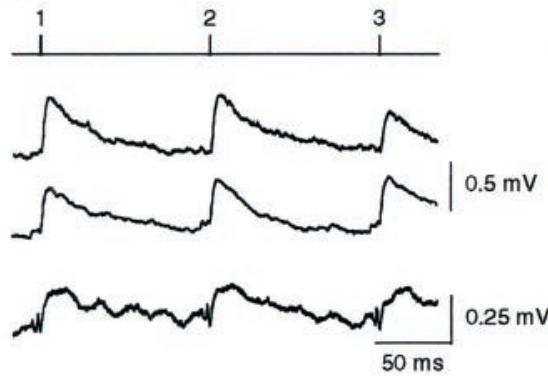

e

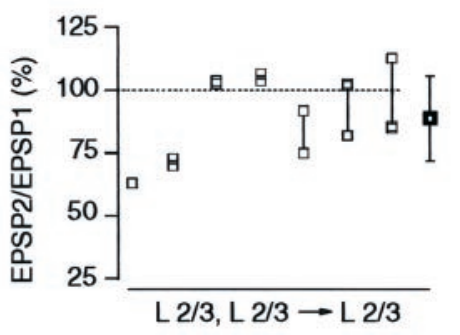

d
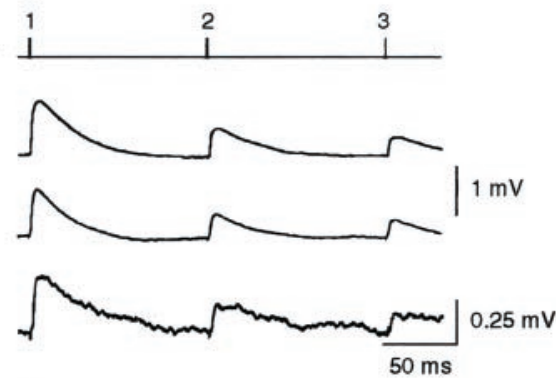

f

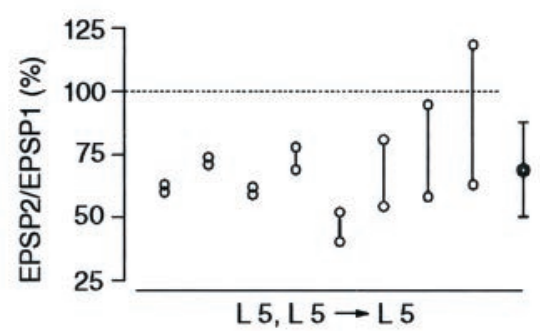

intervals longer than $5 \mathrm{sec}$ to ensure that the amplitudes of the first EPSPs in a train reached control levels. Averages of EPSPs were compiled from 50 to 200 sweeps. After data collection, whole-cell recording was reestablished with pipettes filled with an intracellular solution containing $0.5 \%$ biocytin to label the cells for subsequent morphological reconstruction. Stimulus delivery and data acquisition and analyses were made using macros in IGOR (Wavemetrics, Lake Oswego, OR).

Histological procedures. Slices were stored in $4 \%$ paraformaldehyde for up to 2 weeks. Slices were subsequently processed for biocytin labeling in whole-mount sections using a modified procedure described by Horikawa and Armstrong (1988). After being rinsed with $1 \% \mathrm{H}_{2} \mathrm{O}_{2}$ in $10 \% \mathrm{MeOH}$ and $90 \%$ phosphate buffer $(0.1 \mathrm{M})$, slices were incubated for $1 \mathrm{hr}$ in $2 \%$ Triton X-100 and subsequently exposed to the avidin-biotin peroxidase complex (ABC kit; Vector Laboratories, Burlingame, CA) for $2 \mathrm{hr}$. Slices were rinsed with phosphate buffer and reacted with 3,3-diaminobenzidine (Sigma, St. Louis, MO). Slices were mounted onto slides with Mowiol
(Hoechst Pharmaceuticals, Frankfurt, Germany) and viewed using either a $40 \times$ or $100 \times$ oil immersion objective. The cells were morphologically reconstructed with the Neurolucida system (MicroBrightField, Colchester, VT).

\section{RESULTS}

\section{Identification of pyramidal cells}

Pyramidal cells were identified under IR-DIC (see Materials and Methods) video microscopy by their triangular somata, each of which gave rise to an apical dendrite that extended toward the pial surface. These cells were classified into layer 2/3 (L2/3) and L5 pyramidal cells according to their locations in supra- and infragranular cortex. Reconstructions of representative, biocytin- 
labeled pyramidal neurons in layers $2 / 3$ and 5 (Fig. $1 a, b$ ) show that pyramidal cells in layer 5 were of the thick, tufted type (Markram et al., 1997) and pyramidal cells in layer $2 / 3$ were comparable with those described previously (Mason et al., 1991; Schröder and Luhmann, 1997; Reyes et al., 1998; Thomson and Bannister, 1998). Suprathreshold stimulation of the presynaptic neurons by brief current injection through the soma evoked unitary EPSPs that were blocked by bath application of $30 \mu \mathrm{M} \mathrm{CNQX}$ and $50 \mu \mathrm{M}$ APV, indicating that the synapses were glutamatergic.

\section{Connections within layer $2 / 3$ and within layer 5 of young cortex}

Frequency-dependent modification of unitary EPSPs was first examined in cells from young (P14) cortex. Action potentials triggered by a train $(10 \mathrm{~Hz})$ of two to three brief current pulses into the somata of the presynaptic cells evoked a train of unitary EPSPs in the target pyramidal neurons. In Figure $1 c$, three different presynaptic layer $2 / 3$ neurons were stimulated sequentially (onsets of action potentials depicted by the numbered tic marks in the upper trace) to evoke unitary EPSPs in a fourth layer 2/3 target neuron (three lower traces). The amplitude of the second and third EPSP, in most experiments, decreased only slightly or not at all during the train. In contrast, the EPSPs evoked in layer 5 neurons depressed strongly. Figure $1 d$ shows EPSPs recorded in the same layer 5 neuron during sequential stimulation of two presynaptic layer 5 neurons (second and fourth traces). The EPSPs evoked in another target neuron by stimulation of one of the presynaptic neurons also showed depression (Fig. 1d, third trace). Thus, in contrast to the EPSPs evoked between layer 2/3 neurons, those evoked between layer 5 neurons using the same stimulus protocol depressed strongly. To quantify the degree of short-term modification of EPSPs, we divided the peak amplitude of the second EPSP in the train by that of the first EPSP to obtain the amplitude ratio (EPSP2/EPSP1 $\times 100)$. The mean $( \pm$ SD) amplitude ratio of EPSPs evoked in connected layer 2/3 neurons was $97 \pm 23 \%$ ( $n=44$; see also Fig. 3$)$ and was significantly $(p<$ 0.001 , two-tailed $t$ test) higher than the amplitude ratio of EPSPs evoked in connected layer 5 neurons $(70 \pm 20 \%$; $n=52$; see also Fig. 3).

Simultaneous triple recordings from circuits consisting of two presynaptic neurons that innervated the same target cell revealed that, within each layer, amplitude ratios of EPSPs evoked in the same target neuron were variable. Figure 1 summarizes results from seven triple recordings in layer $2 / 3$ (Fig. 1e) and eight triple recordings in layer 5 (Fig. $1 f$ ). The connected symbols in each graph represent the amplitude ratios of EPSPs evoked in the same target neuron when two, and in some cases three, presynaptic neurons were stimulated individually at $10 \mathrm{~Hz}$. Within either cortical layer, the EPSPs evoked in individual layer 5 neurons tended to depress strongly, whereas those evoked in individual layer $2 / 3$ neurons tended either to depress weakly or to remain relatively unchanged. However, the amplitude ratios of the two EPSPs evoked in a common target could differ by as much as $50 \%$. In two cases, the EPSPs evoked by stimulation of one presynaptic neuron depressed, whereas those evoked in the same neuron after stimulation of the other presynaptic neuron facilitated.

Although the unitary EPSPs evoked in layer 2/3 were smaller $(0.5 \pm 0.5 \mathrm{mV}$, mean $\pm \mathrm{SD} ; n=44)$ than those evoked in layer 5 $(1.0 \pm 0.9 \mathrm{mV} ; n=52)$, the layer-specific differences in the amplitude ratios were independent of the EPSP amplitude, taken as the peak of the first EPSP of the train. In Figure 1c, second and fourth traces, the amplitude of the EPSPs evoked in the same layer
2/3 neuron differed substantially, yet their amplitude ratios were comparable. Similar observations were made in triple recordings from layer 5 (e.g., Fig. 1d, second and fourth traces). For EPSPs evoked in layer $2 / 3$ neurons, a linear regression fit applied to a plot of EPSP amplitude ratio versus EPSP amplitude (data not shown) did not show a significant correlation (slope, $-11.0 \% / \mathrm{mV}$; $\left.r^{2}=0.05\right)$. For EPSPs evoked in layer 5 connections, the slope was $-6.1 \% / \mathrm{mV}\left(r^{2}=0.07\right)$. When the comparison was limited to a small range of EPSP amplitudes $(<0.5 \mathrm{mV})$, the difference in the mean amplitude ratios of EPSPs evoked between layer $2 / 3$ neurons $(n=23)$ remained significantly $(p<0.001)$ different from those evoked between layer 5 neurons $(n=19)$.

\section{Connections between layer 2/3 and layer 5 neurons of young cortex}

To compare directly short-term modification of EPSPs evoked when the pre- and postsynaptic neurons were located in the two different cortical layers, we recorded from circuits in which a layer $2 / 3$ neuron was presynaptic to a layer 5 target neuron. The mean ( \pm SD) amplitude of the first EPSP of the train was $0.3 \pm 0.3 \mathrm{mV}$ $(n=55)$. As with EPSPs evoked in postsynaptic layer $2 / 3$ neurons, the EPSPs evoked in postsynaptic layer 5 neurons depressed weakly with a mean EPSP amplitude ratio of $90 \pm 25 \%(n=55$; see also Fig. 3). Connections where layer 5 pyramidal neurons were presynaptic to layer $2 / 3$ neurons were not found.

To determine the variation of short-term modification of the EPSPs evoked in layer 5 neurons, we recorded simultaneously from convergently connected neurons in which two or more layer $2 / 3$ neurons innervated the same layer 5 neuron (Fig. 2a,c). In Figure $2 e$, the amplitude ratios of EPSPs evoked in the same layer 5 neuron after stimulation of between two to eight different presynaptic layer $2 / 3$ neurons are summarized. Amplitude ratios varied considerably, and sometimes both facilitating and depressing EPSPs were evoked in the same neuron. The degree of variability was most clearly seen in one experiment in which eight EPSPs were evoked in the same layer 5 target neuron after stimulation of eight different presynaptic neurons (Fig. 2e). Similar variability occurred in the amplitude ratios of EPSPs evoked in divergently connected neurons, in which the same layer $2 / 3$ neuron innervated two layer 5 neurons (Fig. $2 b, d$ ). Again in some cases, EPSPs evoked in one target neuron facilitated, whereas those evoked in the other depressed. The fact that the variability of amplitude ratios was comparable for both convergent and divergent connections indicated that individual differences could not be accounted for by specific connections between distinct subpopulations of presynaptic layer $2 / 3$ or postsynaptic layer 5 neurons.

The EPSP amplitude ratios did not vary systematically with the location of putative synaptic contacts, identified under light microscopy, on the layer 5 neuron. Unlike the contacts between layer 5 neurons, which are primarily formed on the basal dendrites (Markram et al., 1997), the putative synaptic contacts formed by layer $2 / 3$ neurons on the layer 5 neurons were primarily on the distal apical trunk or proximal oblique dendrites of the layer 5 neurons $(n=9)$. Typically, the axonal arbor of a layer $2 / 3$ neuron made between one and five putative contacts. In Figure $2 a$, the contacts formed by both presynaptic neurons were on the distal apical dendrites of the layer 5 neuron, yet one input facilitated and the other depressed. Similarly, in Figure $2 b$, the EPSPs evoked in the two target neurons both depressed, although the contact on one target neuron was located distally on the apical 
Figure 2. EPSPs evoked in layer 5 pyramidal neurons by stimulation of layer $2 / 3$ pyramidal neurons. $a$, Camera lucida reconstructions of three pyramidal neurons from a P14 rat in which two layer $2 / 3$ pyramidal neurons innervated a common layer 5 pyramidal neuron, as indicated in the schematic drawing (inset). $b$, Camera lucida reconstructions of three pyramidal neurons from a P14 rat in which a layer $2 / 3$ neuron innervated two layer 5 neurons, as indicated in the schematic drawing (inset). $c$, Unitary EPSPs evoked in the same layer 5 neuron when two presynaptic neurons located in layer $2 / 3$ were stimulated sequentially at $10 \mathrm{~Hz}$. The stimulation pattern is indicated above each voltage record. The resting membrane potential of the postsynaptic neuron was $-61 \mathrm{mV}$. $d$, Unitary EPSPs evoked simultaneously in two layer 5 neurons when a single presynaptic layer $2 / 3$ neuron was stimulated at $10 \mathrm{~Hz}$. Resting potentials of the postsynaptic cells were -58 and -59 $\mathrm{mV}$. $e$, Summary of the amplitude ratios (EPSP2/ EPSP $1 \times 100)$ of EPSPs recorded in layer 5 target neurons. Connected circles represent the amplitude ratios of EPSPs evoked by stimulation of two or more layer $2 / 3$ neurons. The mean $( \pm \mathrm{SD})$ amplitude ratio (shown on the right) is $94 \pm 29 \%(n=23)$. $f$, Summary of the amplitude ratios of EPSPs evoked simultaneously in two layer 5 neurons during stimulation of a single presynaptic layer $2 / 3$ neuron. The mean ( \pm $\mathrm{SD}$ ) amplitude ratio (shown on the right) is $96 \pm 26 \%$ $(n=8)$. a
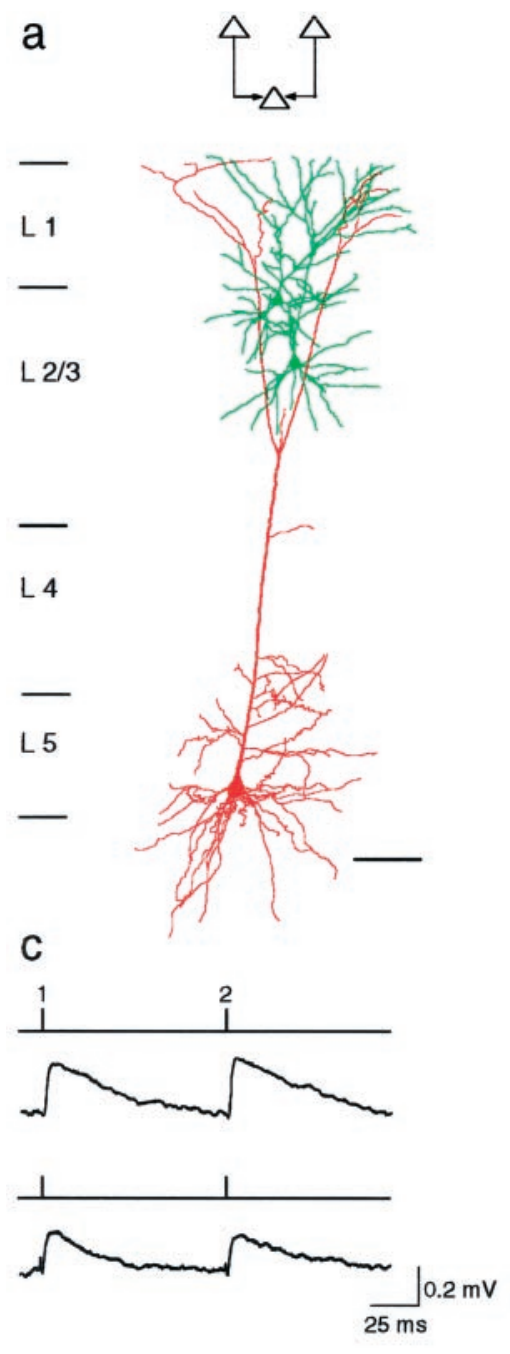

e

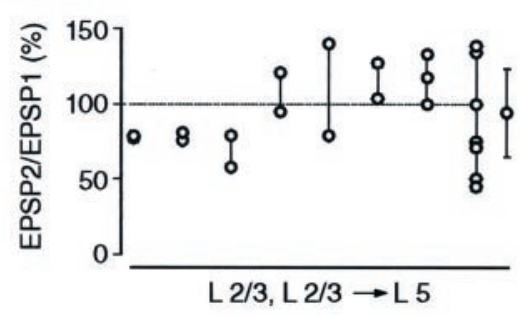

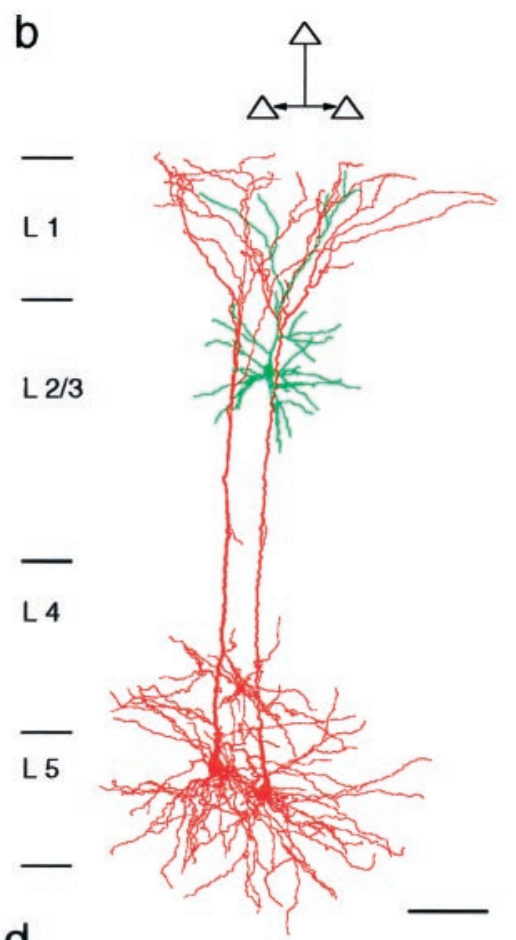

d
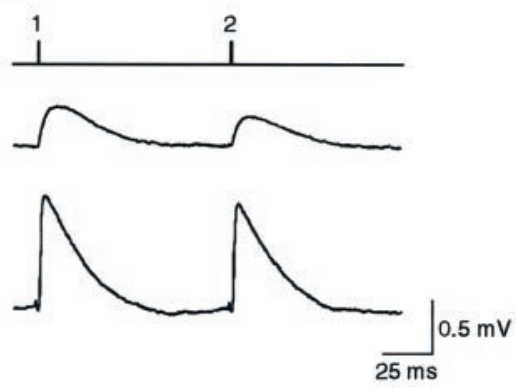

f

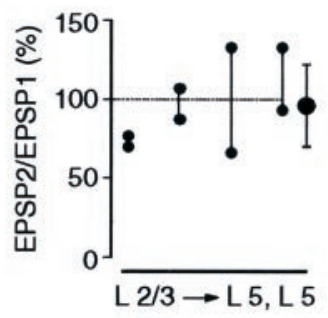

dendrite whereas the contacts on the other were on the proximal oblique and apical dendrites.

The distributions of amplitude ratios for the three different patterns of intra- and interlaminar connections between pyramidal neurons, shown schematically in Figure $3 a$, are summarized in Figure $3 b$. When the presynaptic cells were in layer $2 / 3$, the amplitude ratios of EPSPs evoked in different target neurons (i.e., layer $2 / 3$ or 5 ) were comparable (Fig. $3 b$, top, middle). Stimulation of presynaptic layer $2 / 3$ neurons evoked EPSPs in other layer $2 / 3$ neurons whose mean amplitude ratio $(97 \pm 23 \% ; n=44)$ was not significantly ( $p>0.1$, two-tailed $t$ test) different from that of EPSPs evoked in layer 5 neurons $(90 \pm 25 \%$; $n=55)$. In contrast, the amplitude ratios of EPSPs evoked in layer 5 neurons by stimulation of presynaptic layer $2 / 3$ neurons were significantly $(p<0.001)$ higher than those of EPSPs evoked by stimulation of presynaptic layer 5 neurons (Fig. $3 b$, bottom; $70 \pm 20 \%$; $n=52$ ). Thus, in young (P14) cortex, short-term modification of EPSPs in connections between pyramidal neurons depended predominantly on whether the presynaptic neuron was located in layer $2 / 3$ or layer 5 .

\section{Combined inter- and intralaminar connections}

To confirm further that the degree of depression of EPSPs in young animals was determined predominantly by the layer in which the presynaptic neuron was located, we recorded simultaneously from three divergently connected neurons in which the same layer $2 / 3$ neuron innervated both a layer $2 / 3$ and a layer 5 neuron (Fig. $4 a$, inset). Alternatively, we recorded from convergently connected neurons in which the same layer 5 neuron was 
a

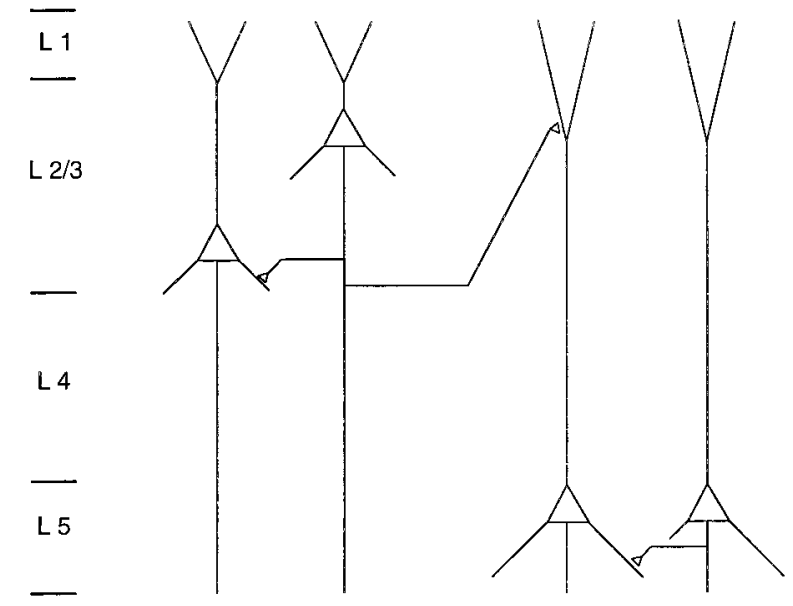

$b$

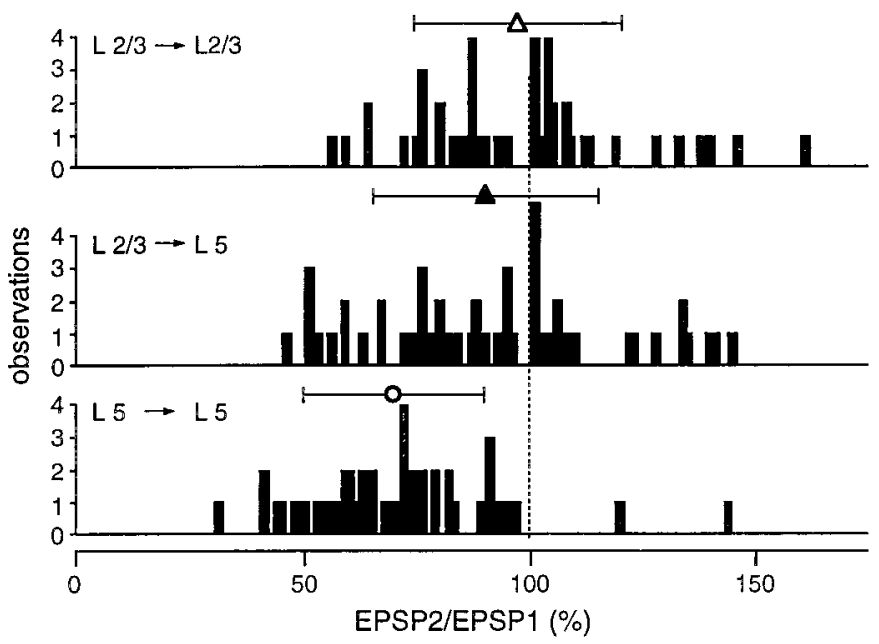

Figure 3. Summary of short-term modification of unitary EPSPs evoked in layer $2 / 3$ and layer 5 pyramidal neurons of young rats. $a$, Schematic drawing of pyramidal cell connectivity patterns examined by simultaneous double and triple whole-cell recordings from pyramidal neurons located in layers $2 / 3$ and 5. $b$, Distributions of EPSP amplitude ratios for unitary EPSPs evoked in connections between layer 2/3 neurons (top), between presynaptic layer $2 / 3$ and postsynaptic layer 5 neurons (middle), and between neurons located in layer 5 (bottom) of P14 rats. Symbols above histograms give the mean ( $\pm \mathrm{SD})$ amplitude ratios for each type of connection. The respective values were $97 \pm 23 \%(n=44$; open triangle $)$, $90 \pm 25 \%(n=55 ;$ filled triangle $)$, and $70 \pm 20 \%(n=52 ;$ open circle $)$. Amplitude ratios for EPSPs evoked between layer 2/3 neurons were not significantly different ( $p>0.1, t$ test) from those evoked between layer $2 / 3$ and layer 5 neurons. Both were significantly $(p<0.001)$ greater than those for EPSPs evoked between layer 5 neurons.

innervated by a presynaptic layer $2 / 3$ and a presynaptic layer 5 neuron (Fig. 4c, inset).

Recordings from divergent triplets showed that the layer $2 / 3$ neurons that innervated other layer $2 / 3$ neurons were from the same population as those that innervated layer 5 neurons. Figure $4 a$ shows EPSPs evoked simultaneously in a layer $2 / 3$ and a layer 5 target neuron during stimulation of a presynaptic layer $2 / 3$ neuron. The amplitude ratios of EPSPs evoked in these divergent triplets are summarized in Figure $4 b$. The EPSPs evoked in layer
$2 / 3$ neurons (Fig. $4 b$, open squares) were not significantly ( $p>$ 0.05 , paired two-tailed $t$ test; $n=7$ triplets) different from those evoked simultaneously in layer 5 neurons ( filled circles).

Recordings from convergently connected triplets indicated that layer $2 / 3$ and layer 5 neurons projected to the same population of layer 5 neurons; hence, layer specificity was not attributable to different presynaptic cells innervating different classes of layer 5 pyramidal cells. In the same target neuron, EPSPs evoked during stimulation of a presynaptic layer $2 / 3$ neuron consistently depressed less than did those evoked during stimulation of a presynaptic layer 5 neuron (Fig. 4c). The amplitude ratios of EPSPs evoked in these convergent triplets are summarized in Figure $4 d$. The amplitude ratios of EPSPs evoked in a common layer 5 neuron during stimulation of layer $2 / 3$ neurons (Fig. $4 d$, filled circles) were significantly ( $p<0.05$, paired $t$ test; $n=9$ triplets) greater than those evoked during stimulation of layer 5 neurons (open circles), confirming that short-term modification of EPSPs in pyramid-to-pyramid connections in the young (P14) cortex depended predominantly on whether the presynaptic neuron was located in layer $2 / 3$ or in layer 5 .

\section{Developmental "switch" from synaptic depression to facilitation}

During development of the brain, the neurons in different cortical layers migrate and mature from the deeper to the upper layers, resulting in a gradient in the degree of maturity (Angevine and Sidman, 1961; Berry and Rogers, 1965). To determine whether the layer-specific differences reflected different stages of development of synaptic contacts, we recorded from pairs of pyramidal neurons in cortical layers $2 / 3$ and 5 of more mature (P18-P28) rats.

Unlike those in P14 cortex, the unitary EPSPs in P28 cortex tended to exhibit paired-pulse facilitation in all connections, independent of the location of the presynaptic neuron. This switch in short-term modification of EPSPs during development was largest in connections between L5 pyramidal neurons. In Figure 5, unitary EPSPs evoked between two connected layer 5 neurons in the P14 cortex (Fig. 5a) are compared with EPSPs evoked in layer 5 neurons in the P28 cortex (Fig. $5 b$ ). The presynaptic neurons were stimulated at 10,20 , and $40 \mathrm{~Hz}$. In the P14 cortex, the EPSPs depressed strongly, whereas in the P28 cortex, the EPSPs facilitated. The mean ( \pm SD) amplitude ratio was $121 \pm 22 \%(n=9)$ at $10 \mathrm{~Hz}$ stimulation. Figure $5 c$ illustrates the time course of the switch in short-term modification of EPSPs from depression to facilitation at four stages of postnatal development. The EPSP amplitude did not consistently continue to increase with successive stimuli. On the average, the amplitude ratio of the third to the first EPSP was $104 \pm 25 \%(n=9)$. This value was nevertheless greater than that for EPSPs evoked in young cortex.

EPSPs evoked between layer 2/3 neurons and between layer 2/3 and layer 5 neurons also became more facilitatory during development. In P28 cortex, the mean ( \pm SD) amplitude ratio for EPSPs evoked between layer $2 / 3$ pyramidal cells was $122 \pm 25 \%$ $(n=8)$, and that for connections between layer $2 / 3$ and layer 5 pyramidal cells was $117 \pm 31 \%(n=7)$. Figure $5 d$ summarizes the mean amplitude ratios for EPSPs evoked in P14 (filled bars) and P28 (open bars) neurons when the presynaptic neurons were stimulated at $10 \mathrm{~Hz}$. For all types of connections, the EPSP amplitude ratios in P28 cortex indicated facilitation and were significantly ( $p \leq 0.01$, two-tailed $t$ test) higher than those of P14 cortex. For EPSPs evoked between layer $2 / 3$ neurons, the ampli- 
Figure 4. EPSPs evoked simultaneously in layer $2 / 3$ and layer 5 pyramidal neurons of young (P14) rats. $a$, A layer $2 / 3$ pyramidal cell innervating both a layer 5 pyramidal cell and another layer $2 / 3$ pyramidal cell, as indicated in the schematic drawing (inset). Stimulation of the presynaptic neuron at $10 \mathrm{~Hz}$ simultaneously evoked EPSPs in the layer $2 / 3$ neuron (upper trace) and in the layer 5 neuron (lower trace). The stimulation pattern is shown above the voltage traces. Resting membrane potentials were $-61 \mathrm{mV}$ for the postsynaptic layer $2 / 3$ neuron and $-62 \mathrm{mV}$ for the postsynaptic layer 5 neuron. $b$, Summary of amplitude ratios of EPSPs evoked simultaneously in layer 2/3 (open squares) and layer 5 ( filled circles) neurons during stimulation of the same presynaptic layer $2 / 3$ neuron. Mean $( \pm$ SD) amplitude ratios (shown on the right) for EPSPs evoked in layer $2 / 3$ and layer 5 neurons were $96 \pm 37 \%$ (open square) and $90 \pm 20 \%$ (filled circle), respectively. $c$, A layer $2 / 3$ pyramidal neuron and a layer 5 neuron innervating the same layer 5 neuron, as indicated in the schematic drawing (inset). Stimulation of either the presynaptic layer $2 / 3$ neuron (upper trace) or the layer 5 neuron (lower trace) at $10 \mathrm{~Hz}$ evoked EPSPs in the layer 5 target neuron. $d$, Summary of amplitude ratios for EPSPs recorded in layer 5 neurons during sequential stimulation of layer 2/3 (filled circles) and layer 5 neurons (open circles). Mean ( \pm SD) amplitude ratios (shown on the right) were $93 \pm 15 \%(n=9$; filled circle) and $73 \pm 16 \%(n=9$; open circle $)$ for EPSPs evoked during stimulation of presynaptic layer $2 / 3$ and layer 5 neurons, respectively. a

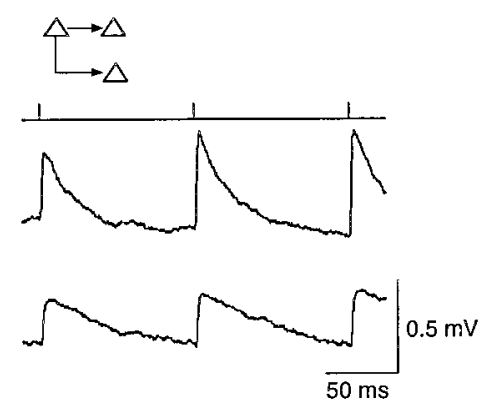

$b$

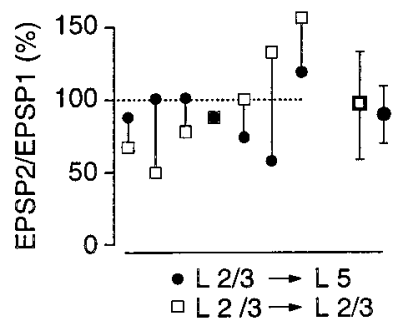

C
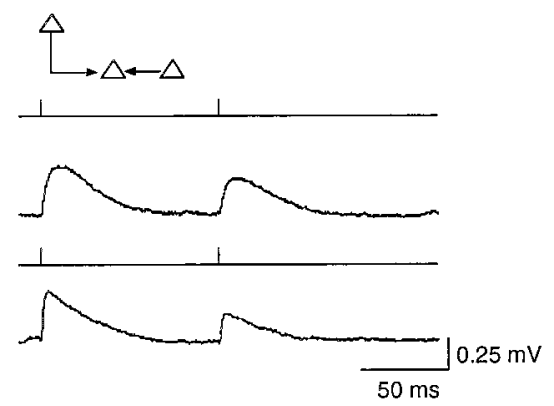

d

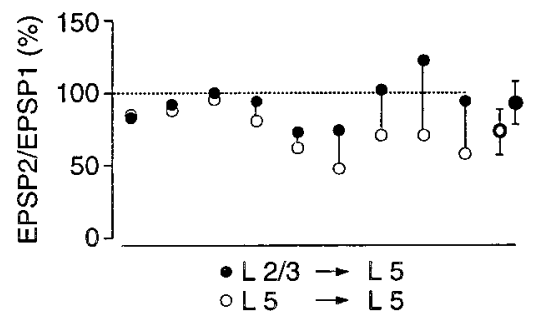

tudes, on the average, continued to increase for the third stimuli; the amplitude ratio of the third to the first EPSP was $138 \pm 57 \%$ $(n=8)$. For EPSPs evoked between layer 2/3 and layer 5 neurons, the amplitude ratio of the third to the first EPSP was $100 \pm 33 \%$ $(n=6)$.

Another difference between P14 and P28 cortices was that the amplitude of unitary EPSPs evoked in the more mature animals tended to be smaller. The mean amplitude of unitary EPSPs evoked in connected P28 layer 5 neurons was $0.3 \pm 0.1 \mathrm{mV}(n=$ 10 ) and was significantly ( $p<0.02$, two-tailed $t$ test) smaller than that for EPSPs evoked in P14 layer 5 neurons $(1.0 \pm 0.9 \mathrm{mV} ; n=$ 52). Similarly, the amplitudes of EPSPs evoked in connections between P28 layer $2 / 3$ neurons $(0.3 \pm 0.1 ; n=8)$ and between layer $2 / 3$ and layer 5 neurons $(0.1 \pm 0.1 \mathrm{mV} ; n=7)$ were smaller than those for EPSPs evoked in P14 cortex (layer 2/3 to layer 2/3, $0.5 \pm 0.5 \mathrm{mV} ; n=44$; layer $2 / 3$ to layer $5,0.3 \pm 0.3 \mathrm{mV} ; n=55)$, although these differences were not significant.

The differences in EPSP amplitudes could not, however, account for the differences in amplitude ratios for the EPSPs evoked in young and mature neurons. For both young (P14) and mature (P28) cortices, a plot of EPSP amplitude ratios versus EPSP sizes revealed no significant correlation in any of the types of connections (data not shown). Furthermore, comparison of the amplitude ratios for EPSPs evoked in young and mature animals when the EPSP sizes were restricted to comparable ranges revealed significant differences for all connections $(p<0.05$, two-tailed $t$ tests).

Presynaptic mechanisms seem to underlie the switch from paired-pulse depression to paired-pulse facilitation. As was done previously (Reyes et al., 1998), the number of times the presynaptic action potentials failed to evoke an EPSP during a stimulus train was quantified (data not shown). In all cases, the EPSP amplitudes were inversely correlated with the failure rates during the train. For depressing EPSPs evoked between layer 5 neurons in young cortex, the decrease in EPSP amplitudes was associated with a progressive increase in the number of failures with each successive stimuli during the train $(n=5)$. For nondepressing EPSPs evoked between layer $2 / 3$ neurons $(n=5)$ and between layer $2 / 3$ and layer 5 neurons $(n=5)$, the number of failures did not change significantly with successive stimuli. For facilitating EPSPs evoked in P28 cortex, the failure rate decreased progressively during the train $(n=5)$. Thus, changes in transmitter release probabilities contributed to short-term modification of EPSPs in both young and more mature cortex.

\section{DISCUSSION}

Frequency-dependent short-term modification of unitary EPSPs was examined in synaptically connected pyramidal neurons in layers $2 / 3$ and 5 of sensorimotor cortex at different stages of postnatal development. In young cortex (P14), EPSPs evoked in all types of connections exhibited, on average, synaptic depression. The degree of depression was determined primarily by the location of the presynaptic cell. When the presynaptic cell was in layer 5, the unitary EPSPs evoked in the target cells exhibited the strongest synaptic depression. In the more mature cortex (P28), the EPSPs on average facilitated weakly regardless of the location of the pre- or the postsynaptic pyramidal cell. Thus, during the third and fourth week of the rat's postnatal development, shortterm modification of EPSPs switched from synaptic depression to facilitation. The switch was particularly striking in the connections between layer 5 pyramidal cells.

The majority of the EPSPs were evoked during $10 \mathrm{~Hz}$ stimulation of the presynaptic cells. Short-term modification of EPSPs was not systematically examined using higher frequency or longer duration stimulus trains to prevent long-term changes in EPSP amplitudes and to minimize temporal summation of EPSPs that could lead to changes in the activation states of ionic conductances. Although there is clear paired-pulse facilitation of EPSPs evoked in P28 cortex, the degree of short-term modification of EPSPs is likely to vary both with the number of successively evoked action potentials and with the stimulus frequency (see Fig. 5) (Markram et al., 1998). 

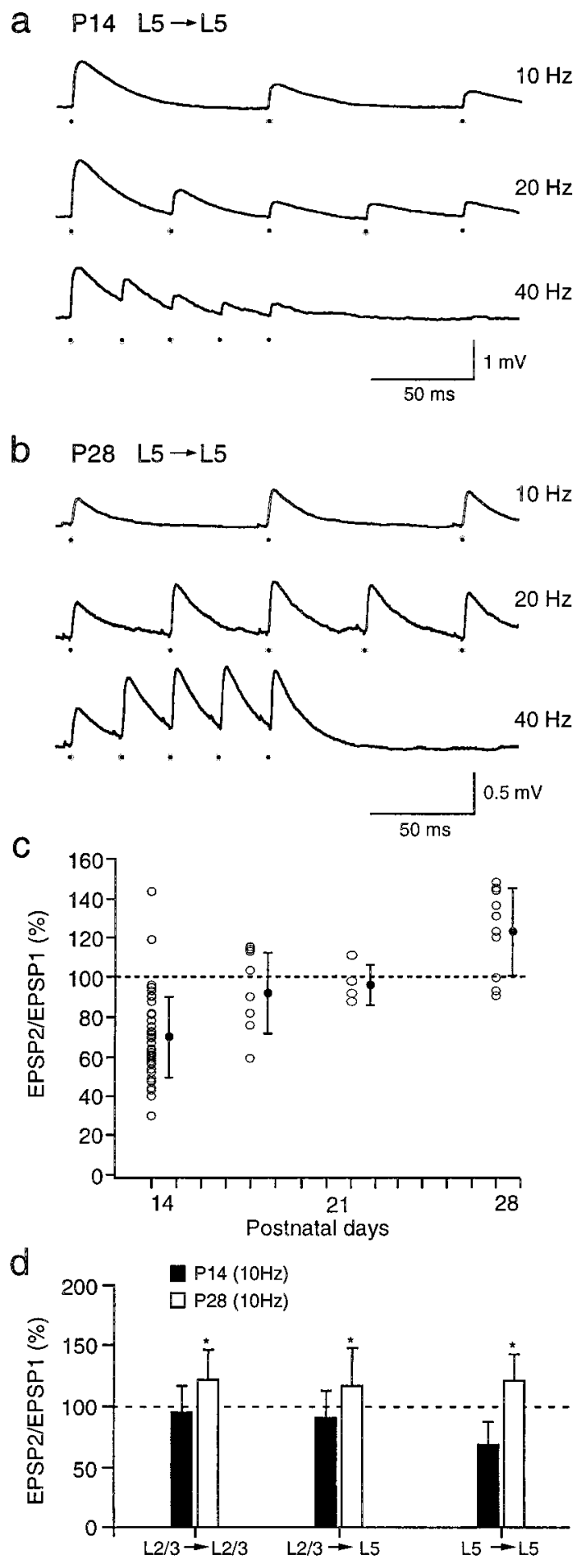

Figure 5. Frequency-dependent short-term modification of EPSPs at different stages of postnatal development. $a$, In young (P14) cortex, stimulation of a presynaptic layer 5 pyramidal cell at 10,20 , and $40 \mathrm{~Hz}$ evoked EPSPs in a postsynaptic layer 5 cell that depressed at all stimulus frequencies. The resting membrane potential was $-68 \mathrm{mV}$. The dots below the voltage traces mark the times of occurrences of presynaptic action potentials. $b$, In more mature (P28) cortex, stimulation of a presynaptic layer 5 pyramidal neuron evoked EPSPs in a postsynaptic layer 5 cell that facilitated at stimulus frequencies of 10,20 , and $40 \mathrm{~Hz}$. $c$, Changes in short-term modification of EPSPs evoked in layer 5 neurons in P14 $(n=$ 52), $\mathrm{P} 18(n=9), \mathrm{P} 22(n=6)$, and $\mathrm{P} 28(n=10)$ rats are shown. The filled circles are means $( \pm \mathrm{SD}) . d$, Comparison of means $( \pm \mathrm{SD})$ of EPSP amplitude ratios for different pyramidal cell connections in layers $2 / 3$ and
Thomson et al. (1993) reported that, on average, EPSPs evoked in layer 5 neurons depressed in "young" adults, although facilitation occurred in at least $21 \%$ of the connections. One source for the discrepancy between these data and those of the present study is that although our recordings were restricted to a homogeneous population of visually identified thick, tufted pyramidal cells in layer 5, their recordings were probably from a more heterogenous population of neurons because cell impalement was made blindly. Indeed, some of their labeled cells were in layer 6 with apical dendrites that did not extend fully to layer 1 . Consistent with this is that their average EPSP amplitude $(1.7 \pm 1.7 \mathrm{mV})$ was considerably larger than that reported here for P28 animals $(0.3 \pm 0.1$ $\mathrm{mV}$ ). Thomson and Bannister (1998) commented also that the EPSPs evoked between layer 3 and layer 5 neurons were depressing.

\section{Projection cell specificity}

Whether frequency-dependent modification of EPSPs is determined by the identity of the pre- or the postsynaptic neuron seems to depend on the particular combination of cell types that are connected. In synapses between principal cells in the olfactory and visual cortex, short-term modification is determined primarily by the identity of the projecting neuron (Bower and Haberly, 1986; Stratford et al., 1996). The results reported here are in agreement with this view because in the young cortex, the degree of EPSP depression seems to be governed by whether the presynaptic neuron was in layer $2 / 3$ or 5 . In contrast, in connections between presynaptic pyramidal cells and postsynaptic nonpyramidal cells in the hippocampus (Ali and Thomson, 1998; Ali et al., 1998) and neocortex (Markram et al., 1998; Reyes et al., 1998), short-term modification of EPSPs is determined by the identity of the target neuron. Similar target specificity was found for EPSPs evoked in motoneurons after stimulation of afferents (Koerber and Mendell, 1991). Despite the target specificity, the mechanism underlying short-term modification of EPSPs seems to be located in the presynaptic terminals (Thomson et al., 1993; Davis and Murphey, 1994; Thomson, 1997; Ali and Thomson, 1998: Ali et al., 1998; Reyes et al., 1998).

\section{Developmental switch from depression to facilitation}

Presynaptically mediated changes in the short-term modification of synaptic efficacy during development have been reported for several types of synaptic connections. Facilitation of EPSPs evoked in hippocampal CA1 pyramidal neurons during stimulation of CA3 afferents increases with age and is accompanied by a decrease in transmitter release probability (Muller et al., 1989; Bolshakov and Siegelbaum, 1995). In the cerebellum, the IPSPs evoked in Purkinje cells undergo a switch from depression to facilitation (Pouzat and Hestrin, 1997). Finally, paired-pulse facilitation of EPSPs evoked in striatal neurons increases substantially at P20 (Choi and Lovinger, 1997). Long-term modification of transmitter release has also been shown to occur only at later stages of development (Ohmori et al., 1981; Pawson and Chase, 1988; Muller et al., 1989; Bolshakov and Siegelbaum, 1995).

$\leftarrow$

5 in P14-P15 ( filled bars) and P28 (open bars) animals is shown. EPSP amplitude ratios were measured during $10 \mathrm{~Hz}$ stimulation of presynaptic cells. Significant ( $p<0.05$, two-tailed $t$ test) differences in the means of the amplitude ratios are marked with asterisks. The number of paired recordings for P14 and P28 animals was, respectively, 44 and 8 for L2/3 to L2/3 connections, 55 and 7 for L2/3 to L5 connections, and 52 and 9 for L5 to L5 connections. 
The switch in the degree of short-term modification of EPSPs coupled with the observation that EPSPs evoked between pyramidal neurons in P28 cortex tended to facilitate could indicate that, in P14 cortex, the synaptic terminals of layer 5 neurons are less mature than are those of layer $2 / 3$ neurons. Because differentiation of neocortical neurons, i.e., arrest of mitosis, proceeds from the deeper, infragranular to the upper, supragranular layers (Angevine and Sidman, 1961; Berry and Rogers, 1965), the axon terminals of pyramidal neurons in each layer may be at different stages of maturation. In particular the terminals of layer 5 neurons might be less differentiated at P14 than at P28, because synaptic connections between layers are already established by precursor cells for the upper layer neurons as the cells pass through layer 5 and migrate to layer $2 / 3$.

\section{Mechanisms underlying layer specificity and the developmental switch}

Short-term modification of EPSPs is based on changes in the probability of evoked transmitter release, suggesting it is mediated predominantly by presynaptic mechanisms (Del Castillo and Katz, 1954; Katz and Miledi, 1968; Betz, 1970; for review, see Zucker, 1989, 1996; Katz, 1996). Consequently, the layer-specific differences in the degree of depression of EPSPs evoked in pyramidal cells at P14 and the developmental switch from depression to facilitation at P28 could be caused primarily by differences in the $\mathrm{Ca}^{2+}$ dynamics in the terminals of the projecting cells. Such differences could involve the $\mathrm{Ca}^{2+}$-mediated component of action potentials in nerve terminals, the mosaic of $\mathrm{Ca}^{2+}$ channel subtypes in the terminals, the $\mathrm{Ca}^{2+}$-binding ratio of the endogenous mobile $\mathrm{Ca}^{2+}$ buffer, or the degree of overlap of $\mathrm{Ca}^{2+}$ domains in active zones. The peak amplitude of somatic action potentials did not change significantly in L5 neurons between P14 and P28, but this does not exclude the possibility that the $\mathrm{Ca}^{2+}$ component of action potentials in terminals is different from that in the soma. The calcium-binding protein calbindin-D28k is expressed stronger in layer $2 / 3$ pyramidal neurons than in layer 5 (Celio, 1990; van Brederode et al., 1991), suggesting that $\mathrm{Ca}^{2+}$ buffering can contribute to layer-specific differences. Alternatively, there may be developmental changes in the release machinery itself, e.g., the $\mathrm{Ca}^{2+}$-binding proteins that constitute the fusion complex (Zucker, 1996).

Postsynaptic conductances do not seem to contribute substantially to the short-term modification of EPSPs. The fact that both facilitation and depression were observed in the same neuron argues against a role for somatic conductances. Furthermore, the stimulus rates were sufficiently low so that the second EPSP did not summate with the first to cause an increased depolarization that could change the activation states of either somatic or dendritic conductances. Finally, when the amplitudes of EPSPs evoked during the train were normalized, the second EPSP superimposed with the first EPSP; changes in the activation states of the conductances would have been manifested either as a decrease or as a increase in the decay rates of successively evoked EPSPs (Thomson and Bannister, 1998). We cannot, however, exclude the possible contribution of desensitization of glutamate receptors.

\section{Functional significance}

During sensory stimulation, layer $2 / 3$ pyramidal cells are excited via spiny stellate cells in layer 4 (Jones and Peters, 1984). Because of frequency-dependent modification of EPSPs, the spread of excitation among the pyramidal neurons will depend on the discharge rate of presynaptic neurons. At P14, the spread of activity within layer 5 would be limited at high discharge rates because substantial depression of EPSPs occurs at frequencies as low as $10 \mathrm{~Hz}$. Intracortical spread of the afferent excitation horizontally within layer $2 / 3$ and vertically from layer $2 / 3$ to layer 5 would be less affected by high discharge rates because EPSPs evoked between presynaptic layer $2 / 3$ neurons and their targets in layer $2 / 3$ and layer 5 exhibit relatively less depression. In P28 cortex, the EPSPs evoked in pyramidal cells within and between the cortical layers, although smaller, facilitate. Consequently, in contrast to the situation in P14 cortex, the maximal spread of excitation would occur when presynaptic cells fire high frequency (e.g., $40 \mathrm{~Hz}$ ) bursts of action potentials. In this respect, it is interesting to note that the tendency of layer 5 neurons to discharge in bursts increases during postnatal development (J. Zhu and B. Sakmann, unpublished observations), suggesting that there are changes in the action potential initiation mechanism that parallel changes in the transmitter release mechanism of layer 5 pyramidal cells.

\section{REFERENCES}

Abbott LF, Varela JA, Sen K, Nelson SB (1997) Synaptic depression and cortical gain control. Science 275:220-224.

Ali AB, Thomson AM (1998) Facilitating pyramid to horizontal oriensalveus interneurone inputs: dual intracellular recordings in slices of rat hippocampus. J Physiol (Lond) 507:185-199.

Ali AB, Deuchars J, Pawelzik H, Thomson AM (1998) CA1 pyramidal to basket and bistratified cell EPSPs: dual intracellular recordings in rat hippocampal slices. J Physiol (Lond) 507:201-217.

Angevine JB, Sidman RL (1961) Autoradiographic study of cell migration during histogenesis of cerebral cortex in the mouse. Nature 192:766-768.

Berry M, Rogers AW (1965) The migration of neuroblasts in the developing cerebral cortex. J Anat 99:691-709.

Betz WJ (1970) Depression of transmitter release at the neuromuscular junction of the frog. J Physiol (Lond) 206:629-644.

Bolshakov VY, Siegelbaum SA (1995) Regulation of hippocampal transmitter release during development and long-term potentiation. Science 269:1730-1734.

Bower JM, Haberly LB (1986) Facilitating and nonfacilitating synapses on pyramidal cells: a correlation between physiology and morphology. Proc Natl Acad Sci USA 83:1115-1119.

Buhl EH, Tamás G, Szilágyi T, Stricker C, Paulsen O, Somogyi P (1997) Effect: number and location of synapses made by single pyramidal cells onto aspiny interneurones of cat visual cortex. J Physiol (Lond) 500:689-713.

Celio MR (1990) Calbindin D-28k and parvalbumin in the rat nervous system. Neuroscience 35:375-475.

Choi MR, Lovinger DM (1997) Decreased probability of neurotransmitter release underlies striatal long-term depression and postnatal development of corticostriatal synapses. Proc Natl Acad Sci USA 94:2665-2670.

Davis GW, Murphey RK (1994) Long-term regulation of short-term transmitter release properties: retrograde signaling and synaptic development. Trends Neurosci 17:9-13.

Del Castillo J, Katz B (1954) Statistical factors involved in neuromuscular facilitation and depression. J Physiol (Lond) 124:574-585.

Goodman CS, Shatz CJ (1993) Developmental mechanisms that generate precise patterns of neuronal connectivity. Cell 72:77-98.

Horikawa K, Armstrong WE (1988) A versatile means of intracellular labelling: injection of biocytin and its detection with avidin conjugates. J Neurosci Methods 25:1-11.

Jones EG, Peters A (1984) Cerebral cortex, Vol 2, Functional properties of cortical cells (Jones EG, Peters A, eds). New York: Plenum.

Katz B (1996) Neural transmitter release: from quantal secretion to exocytosis and beyond. J Neurocytol 25:677-686.

Katz B, Miledi R (1968) The role of calcium in neuromuscular facilitation. J Physiol (Lond) 195:481-492.

Katz LC, Shatz CJ (1996) Synaptic activity and the construction of cortical circuits. Science 274:1133-1138.

Koerber HR, Mendell LM (1991) Modulation of synaptic transmission 
at Ia-afferent fiber connections on motoneurons during high-frequency stimulation: role of postsynaptic target. J Neurophysiol 65:590-597.

Markram H, Tsodyks M (1996) Redistribution of synaptic efficacy between neocortical pyramidal neurons. Nature 382:807-810.

Markram H, Lübke J, Frotscher M, Roth A, Sakmann B (1997) Physiology and anatomy of synaptic connections between thick tufted pyramidal neurones in the developing rat neocortex. J Physiol (Lond) 500:409-440.

Markram H, Wang Y, Tsodyks M (1998) Differential signaling via the same axon of neocortical pyramidal neurons. Proc Natl Acad Sci USA 95:5323-5328.

Mason A, Nicoll A, Stratford K (1991) Synaptic transmission between individual pyramidal neurons of the rat visual cortex in vitro. J Neurosci $11: 72-84$

Muller D, Oliver M, Lynch G (1989) Developmental changes in synaptic properties in hippocampus of neonatal rats. Brain Res 49:105-114.

Ohmori H, Rayport SG, Kandel ER (1981) Emergence of posttetanic potentiation as a distinct phase in the differentiation of an identified synapse in Aplysia. Science 213:1016-1018.

Pawson PA, Chase R (1988) The development of transmission at an identified molluscan synapse. I. The emergence of synaptic plasticities. J Neurophysiol 60:2196-2210.

Pouzat C, Hestrin S (1997) Developmental regulation of basket/stellate cell $\rightarrow$ Purkinje cell synapses in the cerebellum. J Neurosci 17:9104-9112.

Reyes A, Lujan R, Rozov A, Burnashev N, Somogyi P, Sakmann B (1998) Target cell specific facilitation and depression in neocortical circuits. Nat Neurosci 1:279-285.

Schröder R, Luhmann HJ (1997) Morphology, electrophysiology and pathophysiology of supragranular neurons in rat primary somatosensory cortex. Eur J Neurosci 9:163-176.

Stratford KJ, Tarczy-Hornoch K, Martin KAC, Bannister NJ, Jack JJB (1996) Excitatory synaptic inputs to spiny stellate cells in cat visual cortex. Nature 382:258-261.

Stuart GJ, Dodt HU, Sakmann B (1993) Patch clamp recordings from the soma and dendrites of neurones in brain slices using infrared video microscopy. Pflügers Arch 423:511-518.

Thomson AM (1997) Activity-dependent properties of synaptic transmission at two classes of connections made by rat neocortical pyramidal axons in vitro. J Physiol (Lond) 502:131-147.

Thomson AM, Bannister AP (1998) Postsynaptic pyramidal target selection by descending layer III pyramidal axons: dual intracellular recordings and biocytin filling in slices of rat neocortex. Neuroscience 84:669-683.

Thomson AM, Deuchars J (1994) Temporal and spatial properties of local circuits in neocortex. Trends Neurosci 17:119-126.

Thomson AM, Deuchars J, West DC (1993) Large, deep layer pyramidpyramid single-axon EPSPs in slices of rat motor cortex display paired pulse and frequency-dependent depression, mediated presynaptically, and self-facilitation, mediated postsynaptically. J Neurophysiol 70:2354-2369.

Van Brederode JFM, Helliesen MK, Hendrickson AE (1991) Distribution of the calcium-binding proteins parvalbumin and calbindin-D28k in the sensorimotor cortex of the rat. Neuroscience 44:157-171.

Zucker RS (1989) Short term synaptic plasticity. Annu Rev Neurosci 12:13-31.

Zucker RS (1996) Exocytosis: a molecular and physiological perspective. Neuron 17:1049-1055. 